Supporting Information

\title{
Preparation of Surfactant-Resistant Polymersomes with Ultrathick Membranes through RAFT Dispersion Polymerization
}

\author{
Ziyuan Song ${ }^{\dagger}$, Yinzhao Huang ${ }^{\ddagger}$, Vikram Prasad ${ }^{\S}$, Ryan Baumgartner ${ }^{\ddagger}$, Siyi Zhang ${ }^{\dagger}$, Keith \\ Harris", Joshua S. Katz ${ }^{\perp}$, Jianjun Cheng*,† \\ "Department of Materials Science and Engineering, and Department of Chemistry, \\ University of Illinois at Urbana-Champaign, Urbana, Illinois 61801, United States \\ ${ }^{8}$ Home, Institutional and Personal Care Solutions, and "Formulation Science, Corporate \\ Research and Development, The Dow Chemical Company, Midland, Michigan 48667, \\ United States \\ ${ }^{\perp}$ Formulation Science, Corporate Research and Development, The Dow Chemical \\ Company, Collegeville, Pennsylvania 19426, United States
}




\section{Materials}

All chemicals were purchased from Sigma-Aldrich (St. Louis, MO, USA) and used as received unless otherwise specified. Styrene $(\mathrm{St})$ monomer was purified by passing a SDTR-7 inhibitor removal column (Scientific Polymer Products, Inc., Ontario, NY, USA) and stored at $-20{ }^{\circ} \mathrm{C}$. 2,2'-Azobis(2-methylpropionitrile) (AIBN) was purified by recrystallization from ethanol. S-Dodecyl-S'-( $\alpha, \alpha^{\prime}$-dimethyl- $\alpha^{\prime \prime}$-acetic acid) trithiocarbonate terminated methoxy poly(ethylene oxide) (mPEO-DDMAT) macro chain transfer agent (macro-CTA) was synthesized according to the literature procedure. ${ }^{1}$ Carbon film supported copper grids (200 mesh) were purchased from Electron Microscopy Sciences (Hatfield, PA, USA). Silicon wafers $(5 \times 5 \mathrm{~mm})$ were purchased from Ted Pella Inc. (Redding, CA, USA). Spectra/Por RC dialysis tubing with a molecular weight cut-off (MWCO) of $1 \mathrm{kDa}$ was purchased from Spectrum Laboratories (Rancho Dominguez, CA, USA). Pur-A-Lyzer midi dialysis tubes (MWCO $=6 \mathrm{kDa}$ ) were purchased from Sigma-Aldrich (St. Louis, MO, USA). Amicon Ultra-4 centrifugal filters $(\mathrm{MWCO}=10 \mathrm{kDa})$ were purchased from EMD Millipore (Billerica, MA, USA).

Anionic surfactant STEOL CS-370 and nonionic surfactant BIO-SOFT N25-7 were graciously provided by Stepan Company (Elwood, IL, USA). Anionic surfactant DOWFAX 2A1 and nonionic surfactants ECOSURF EH-6, EH-9, and EH-14 were graciously provided by The Dow Chemical Company (Midland, MI, USA).

\section{Instrumentation}

${ }^{1} \mathrm{H}$ NMR spectra were recorded on a Varian U500 spectrometer. Chemical shifts were reported in $\mathrm{ppm}$ and referenced to the solvent proton impurities. Gel permeation chromatography (GPC) experiments were performed on a system equipped with an isocratic pump (Model 1100, Agilent Technology, Santa Clara, CA, USA), a DAWN HELEOS multi-angle laser light scattering detector (MALLS) detector (Wyatt Technology, Santa Barbara, CA, USA), and an Optilab rEX refractive index detector (Wyatt Technology, Santa Barbara, CA, USA). The detection wavelength of HELEOS was set at $658 \mathrm{~nm}$. Separations were performed using serially connected size exclusion columns $\left(10^{2} \AA, 10^{3} \AA, 10^{4} \AA, 10^{5} \AA\right.$, and $10^{6} \AA$ Phenogel columns, $5 \mu \mathrm{m}, 300 \times 7.8 \mathrm{~mm}$, 
Phenomenex, Torrance, CA, USA) at $60{ }^{\circ} \mathrm{C}$ using DMF containing $0.1 \mathrm{~mol} / \mathrm{L} \mathrm{LiBr}$ as the mobile phase. The MALLS detector was calibrated using pure toluene and can be used for the determination of the absolute molecular weights (MWs). The MWs of polymers were determined based on the $\mathrm{dn} / \mathrm{dc}$ value of each polymer sample calculated offline by using the internal calibration system processed by the ASTRA 6 software (version 6.1.1.17, Wyatt Technology, Santa Barbara, CA, USA). Transmission electron microscopy (TEM) images were collected using JEOL 2100 cryo transmission electron microscope. Scanning electron microscopy (SEM) images were recorded with Hitachi S4700 or S4800 scanning electron microscope. Fluorescent spectra were recorded on a PerkinElmer LS 55 fluorescence spectrometer (PerkinElmer, Santa Clara, CA, USA). The turbidity results (UV absorbance at $500 \mathrm{~nm}$ ) were collected on a PerkinElmer Lambda 25 UV/Vis spectrometer (PerkinElmer, Santa Clara, CA, USA). Dynamic light scattering (DLS) results were recorded on a 90Plus particle size analyser (Brookhaven Instruments Coporation, Holtsville, NY, USA).

\section{One-pot Preparation of PEO-b-PSt Polymersome through RAFT Dispersion Polymerization}

PEO- $b$-PSt polymersome was prepared through RAFT dispersion polymerization following the literature procedure. ${ }^{1}$ Typically, mPEO-DDMAT macro-CTA (15.3 mg, 2.9 $\mu \mathrm{mol})$ and St (3 g, $28.8 \mathrm{mmol})$ were dissolved in methanol $(1.8 \mathrm{~mL})$, followed by adding methanol solution of AIBN $(10 \mathrm{mg} / \mathrm{mL}, 47.1 \mu \mathrm{L}, 0.29 \mu \mathrm{mol})$ ([St]:[macro-CTA]:[AIBN] $=10,000: 1: 0.1)$. The resulting solution was then transferred to a $25 \mathrm{~mL}$ Schlenk tube. After three freeze-pump-thaw cycles, the tube was sealed under vacuum. The tube was placed in an oil bath and the polymerization solution was stirred at $80^{\circ} \mathrm{C}$. After $40 \mathrm{~h}$, the polymerization was quenched by cooling down to $\mathrm{rt}$ and exposing to air. The polymersome suspension was purified by dialysis against methanol to remove excessive St $(\mathrm{MWCO}=1 \mathrm{kDa})$, and then exchanged to water medium through ultrafiltration $(\mathrm{MWCO}=10 \mathrm{kDa})$. The obtained aqueous suspension of PEO- $b$-PSt polymersome was ready to use for following characterization and stability studies.

The RAFT dispersion polymerization was also tried at lower temperature $\left(60{ }^{\circ} \mathrm{C}\right)$ to 
better match the decomposition temperature of AIBN. However, the obtained DP of PSt block was only 500 after $40 \mathrm{~h}$ (compared with DP $\sim 1400$ at $80{ }^{\circ} \mathrm{C}$ after $40 \mathrm{~h}$ ) that did not meet our requirement for the design of polymersomes with ultrathick membranes.

In order to encapsulate Rhodamine B (RhB) with PEO-b-PSt polymersome, the methanol solution of RhB ( $2 \mathrm{mg} / \mathrm{mL})$ was used as the solvent. The Schlenk tube was protected by aluminum foil during polymerization to avoid photo-bleaching of $\mathrm{RhB}$ dye. After polymerization and removal of excessive St by dialysis, free RhB dye outside PEO- $b$-PSt polymersome was removed by ultrafiltration $(\mathrm{MWCO}=10 \mathrm{kDa})$ until no fluorescent signal was detected from the washing waste. The obtained aqueous suspension of $\mathrm{RhB}$ loaded PEO- $b$-PSt polymersome was ready to use for following characterization and stability studies. The amount of encapsulated Rhodamine $(\mathrm{RhB})$ was determined by dissolving RhB loaded polymersomes $(100 \mu \mathrm{L})$ with THF $(900 \mu \mathrm{L})$, which was subjected to UV-Vis tests at $\lambda_{\max }=545 \mathrm{~nm}$. The RhB amount was calculated using standard curve of RhB in THF:water co-solvent (9:1, v/v).

\section{PEO-b-PSt Diblock Copolymer Characterization}

An aliquot of the suspension after RAFT dispersion polymerization (before dialysis against $\mathrm{MeOH}$ ) was taken out for the PEO- $b$-PSt diblock copolymers characterization. Typically, the copolymer assemblies was first dissolved by adding THF into the suspension. The resulting clear solution was then precipitated in ether, collected with centrifugation, and washed three times with ether to remove any remaining solvents. The solid polymer residue was dried under vacuum to yield white viscous powder, which was dissolved in $\mathrm{CDCl}_{3}(\sim 5 \mathrm{mg} / \mathrm{mL})$ and DMF $(10 \mathrm{mg} / \mathrm{mL}$, with $0.1 \mathrm{~mol} / \mathrm{L} \mathrm{LiBr})$ for ${ }^{1} \mathrm{H}$ NMR and GPC characterization, respectively.

\section{Preparation of PEO-b-PSt Polymersome through Solvent-Switch Method}

$\mathrm{PEO}_{20}-b-\mathrm{PSt}_{250}$ diblock copolymers were purchased from Sigma-Aldrich (St. Louis, MO, USA) and self-assembled following the literature procedure. ${ }^{2}$ The DMF solution of diblock copolymers $(1 \mathrm{~mL}, 20 \mathrm{mg} / \mathrm{mL})$ was placed in a vial charged with a stir bar. 
Distilled (DI) water $(0.25 \mathrm{~mL})$ was then added to the vial dropwise through a syringe pump (KD Scientific, Holliston, MA, USA. Addition speed: $0.1 \mathrm{~mL} \mathrm{~min}^{-1}$ ). The dispersion was then dialyzed against DI water to remove DMF $(\mathrm{MWCO}=1 \mathrm{kDa})$. The obtained aqueous suspension of PEO- $b$-PSt polymersome with thinner membrane was ready to use for following stability studies.

\section{Transmission Electron Microscopy (TEM) and Scanning Electron Microscopy (SEM)}

TEM samples were prepared on carbon film supported copper grids (200 mesh). One drop $(\sim 10 \mu \mathrm{L})$ of the polymersome aqueous suspension $(0.5 \mathrm{mg} / \mathrm{mL})$ was placed on the grid and allowed to interact with the surface for $10 \mathrm{~min}$. Filter paper was then used to remove the residual polymers and liquid. The sample on the grid was imaged using JEOL 2100 cryo TEM at $200 \mathrm{kV}$.

SEM samples were prepared on silicon wafer. One drop $(\sim 10 \mu \mathrm{L})$ of the polymersome aqueous suspension $(0.5 \mathrm{mg} / \mathrm{mL})$ was placed on the wafer and allowed for drying overnight. The sample was coated with Au-Pd (2.5 $\AA / \mathrm{s}, 30 \mathrm{~s})$ and imaged using Hitachi S4700 or S4800 SEM at $3 \mathrm{kV}$.

\section{Turbidity Tests of PEO- $b$-PSt Polymersome in the Presence of Surfactants}

The aqueous suspension of PEO- $b$-PSt polymersome ( 1 or $2 \mathrm{wt} \%, 0.5 \mathrm{~mL}$ ) was mixed with the aqueous solution of surfactants $(10,20,40$, or $80 \mathrm{wt} \%, 0.5 \mathrm{~mL})$ in a $7 \mathrm{~mL}$ vial. The resulting mixture was sealed to avoid water evaporation and incubated at $\mathrm{rt}$. At different time intervals, the mixture was transferred to the quartz cuvette for turbidity measurement at $500 \mathrm{~nm}$ using UV-Vis spectrometer. After turbidity test, the mixture was transferred back for further incubation and turbidity tests. For the turbidity tests in the presence of shear force, the polymersome dispersion was sonicated by a Model 705 probe sonicator (Fisher Scientific, Pittsburgh, PA, USA) with a 14-s pulse sequence (2-s pulse and 1-s delay, $700 \mathrm{~W}, 50 \%$ amplitude) before mixing with surfactants. 


\section{RhB Release Studies from PEO-b-PSt Polymersome in the Presence of Surfactants}

The aqueous solution of surfactants was prepared by dissolving the surfactants in DI water in a $30 \mathrm{~mL}$ vial with a stir bar $(20 \mathrm{~mL}, 5,10,20,40 \mathrm{wt} \%)$. RhB loaded PEO- $b$-PSt vesicle suspension $(500 \mu \mathrm{L})$ was added into a Pur-A-Lyzer tube $(\mathrm{MWCO}=3.5 \mathrm{kDa})$, and the tube was placed in the vial for release studies at $\mathrm{rt}$ with the protection of aluminum foil (to avoid photo-bleaching of RhB). At different time intervals, an aliquot of solution outside Pur-A-Lyzer tube was taken out for fluorescence test $\left(\lambda_{\mathrm{ex}}=545 \mathrm{~nm}, \lambda_{\mathrm{em}}=578.5\right.$ $\mathrm{nm})$. After fluorescence test, the solution was transferred back to the $30 \mathrm{~mL}$ vial for further release studies. The RhB release percentage was calculated using standard curves of $\mathrm{RhB}$ in corresponding aqueous solution of surfactants. For the $\mathrm{RhB}$ release tests in the presence of shear force, the polymersome dispersion was sonicated by a Model 705 probe sonicator (Fisher Scientific, Pittsburgh, PA, USA) with a 14-s pulse sequence (2-s pulse and 1-s delay, $700 \mathrm{~W}, 50 \%$ amplitude) before mixing with surfactants.

\section{References}

(1) Huang, C.-Q.; Pan, C.-Y. Polymer 2010, 51, 5115-5121.

(2) Yu, K.; Eisenberg, A. Macromolecules 1998, 31, 3509-3518.

(3) Kawaguchi, S.; Ito, K., Dispersion polymerization. In Polymer Particles, Okubo, M., Ed. 2005; Vol. 175, pp 299-328.

(4) Kuehne, A. J. C.; Gather, M. C.; Sprakel, J. Nat. Commun. 2012, 3, 1088. 

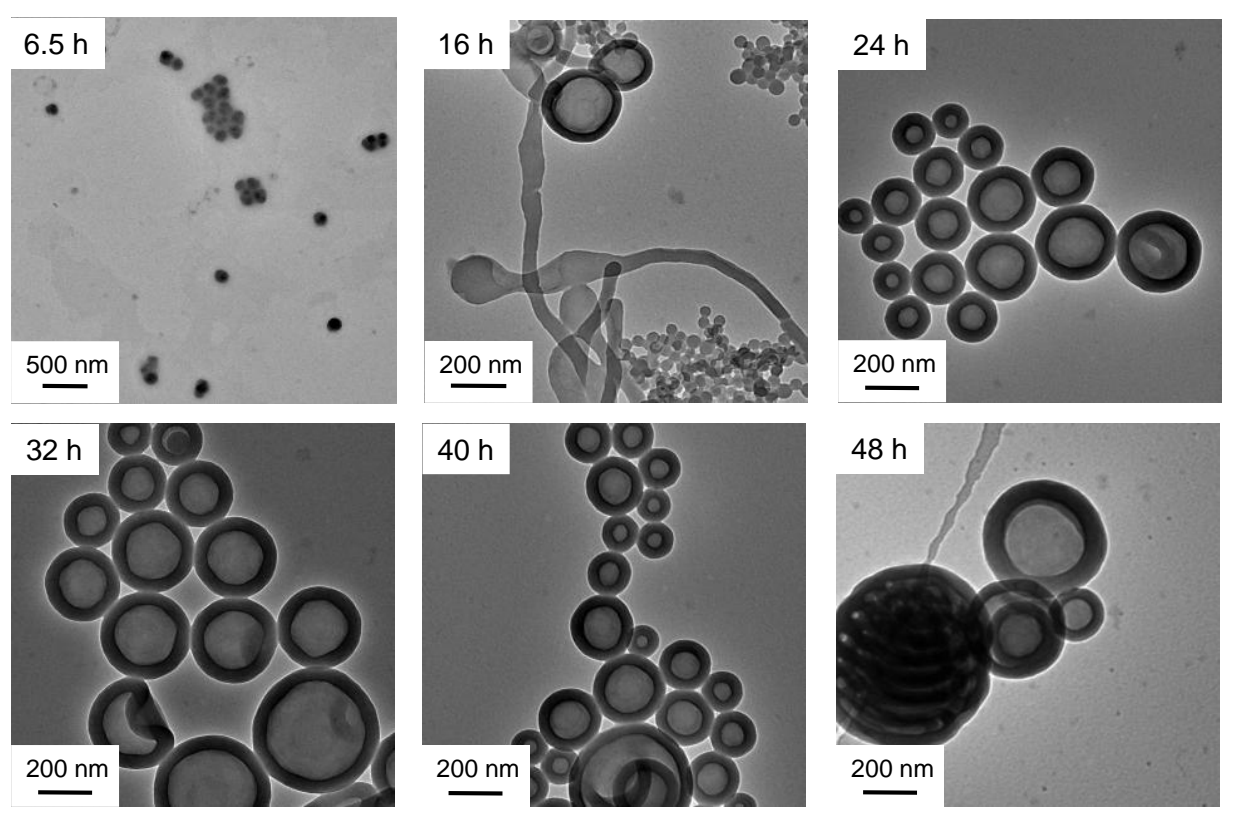

Figure S1. TEM images of PEO- $b$-PSt self-assemblies after different polymerization times.

No self-assembled structures observed for 2-h RAFT dispersion polymerization batch.

Since the size of assemblies is largely controlled by the instant at which stericallystabilized particles form for polymerization-induced self-assembly (PISA) system, ${ }^{3}$ we suggest the formation of sterically-stabilized particles occur at different time in the RAFT dispersion polymerization due to the high fraction of monomers. The non-synchronized sterically-stabilized particle formation leads to broad particle distributions. Such highconcentration-induced particle distribution was also reported in another dispersion polymerization system. ${ }^{4}$ 

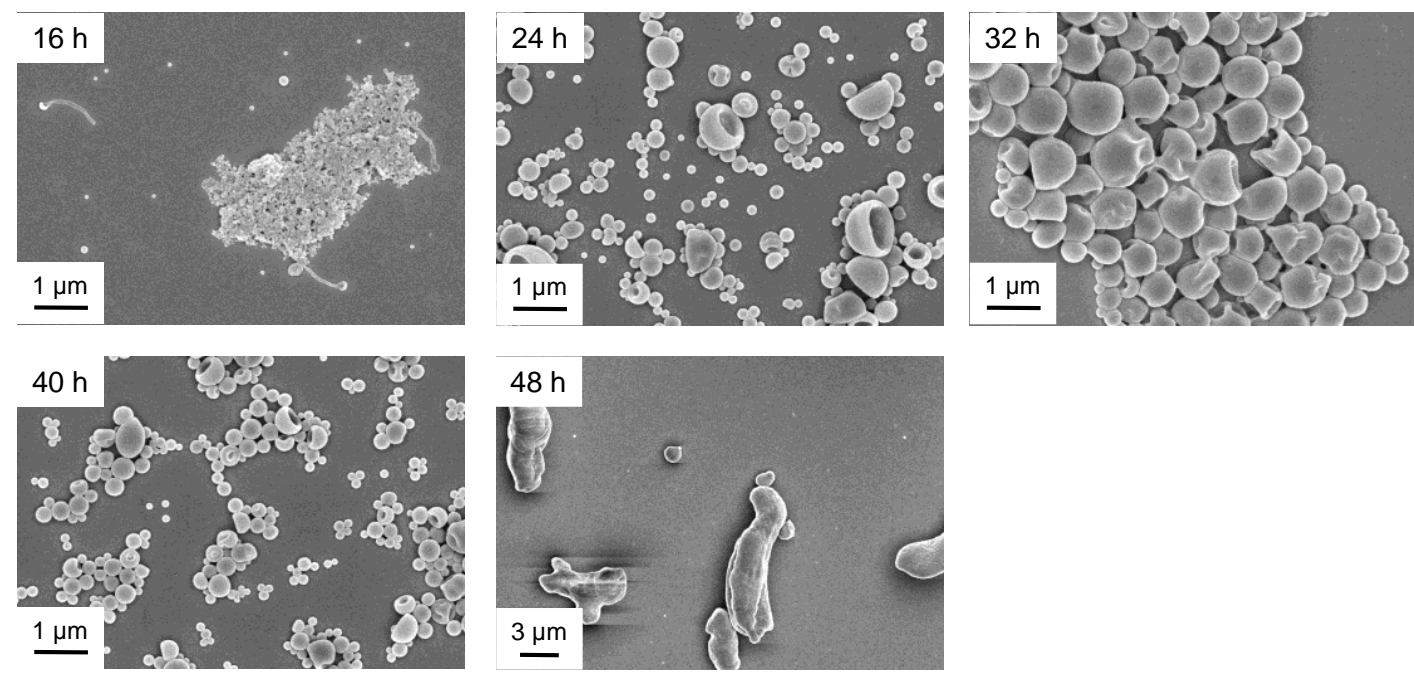

Figure S2. SEM images of PEO- $b$-PSt self-assemblies after different polymerization times.

No self-assembled structures observed for 2-h RAFT dispersion polymerization batch. The micelle morphology from 6.5-h RAFT dispersion polymerization batch was not clear in SEM images and was not shown here. 


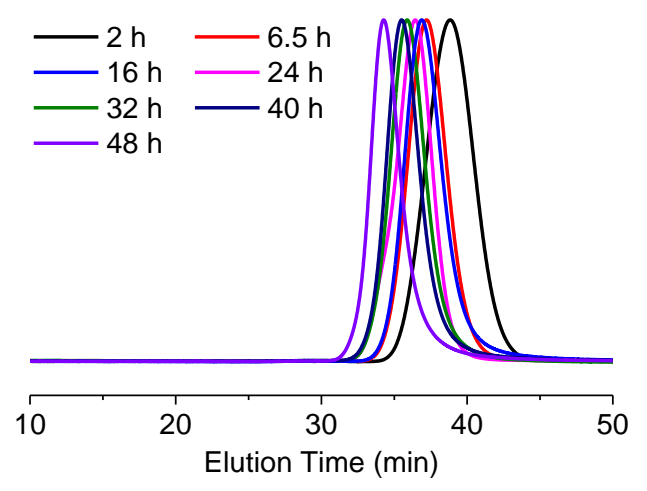

Figure S3. GPC traces of PEO- $b$-PSt after different polymerization times.

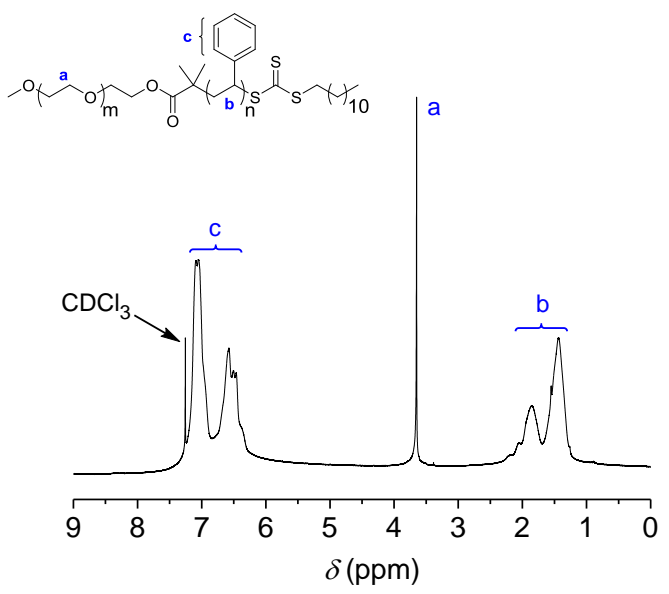

Figure S4. Representative ${ }^{1} \mathrm{H}$ NMR spectrum of PEO- $b$-PSt in $\mathrm{CDCl}_{3}$.

The composition of PEO- $b$-PSt diblock copolymers was calculated from the integral ratio of proton $\mathrm{b}$ (the backbone protons from PSt block) to proton $a$ (methylene protons from PEO block), where the degree of polymerization (DP) of PEO was known as 113 (molecular weight $=5 \mathrm{kDa})$. 

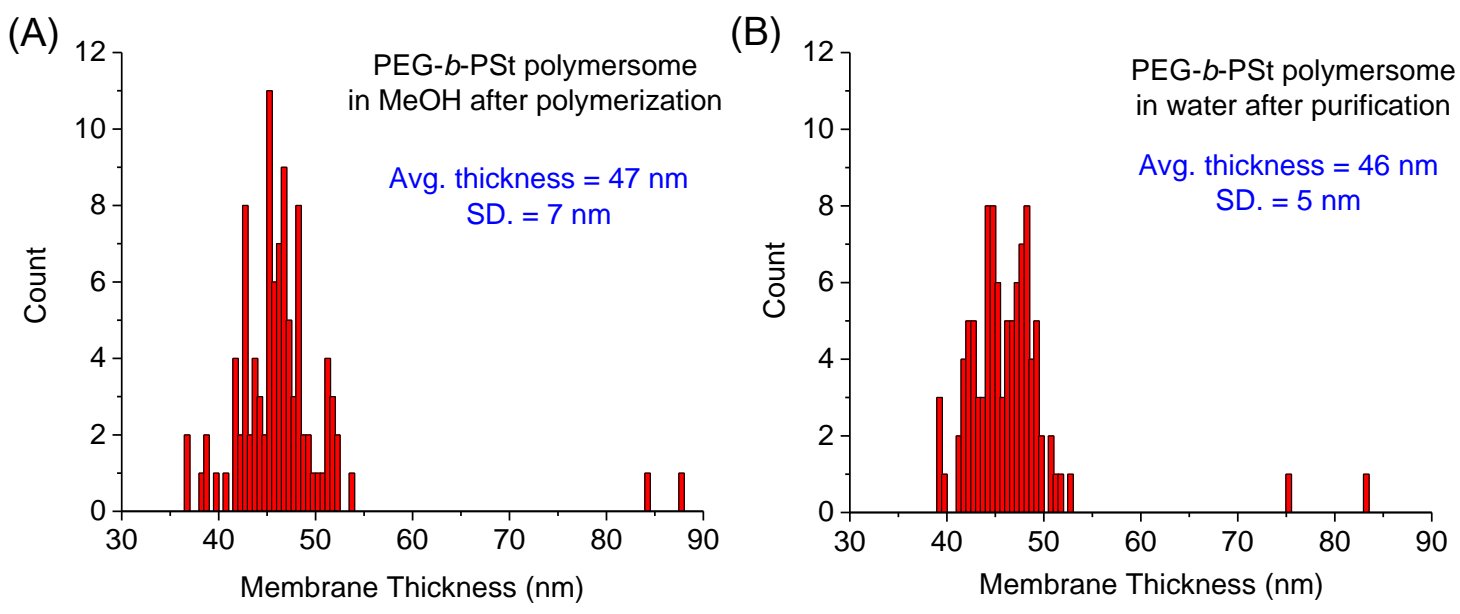

Figure S5. Membrane thickness distribution in methanol after RAFT dispersion polymerization (A) and in water after purification (B). The membrane thickness was measured by counting 100 polymersomes selected from 20 TEM images. 

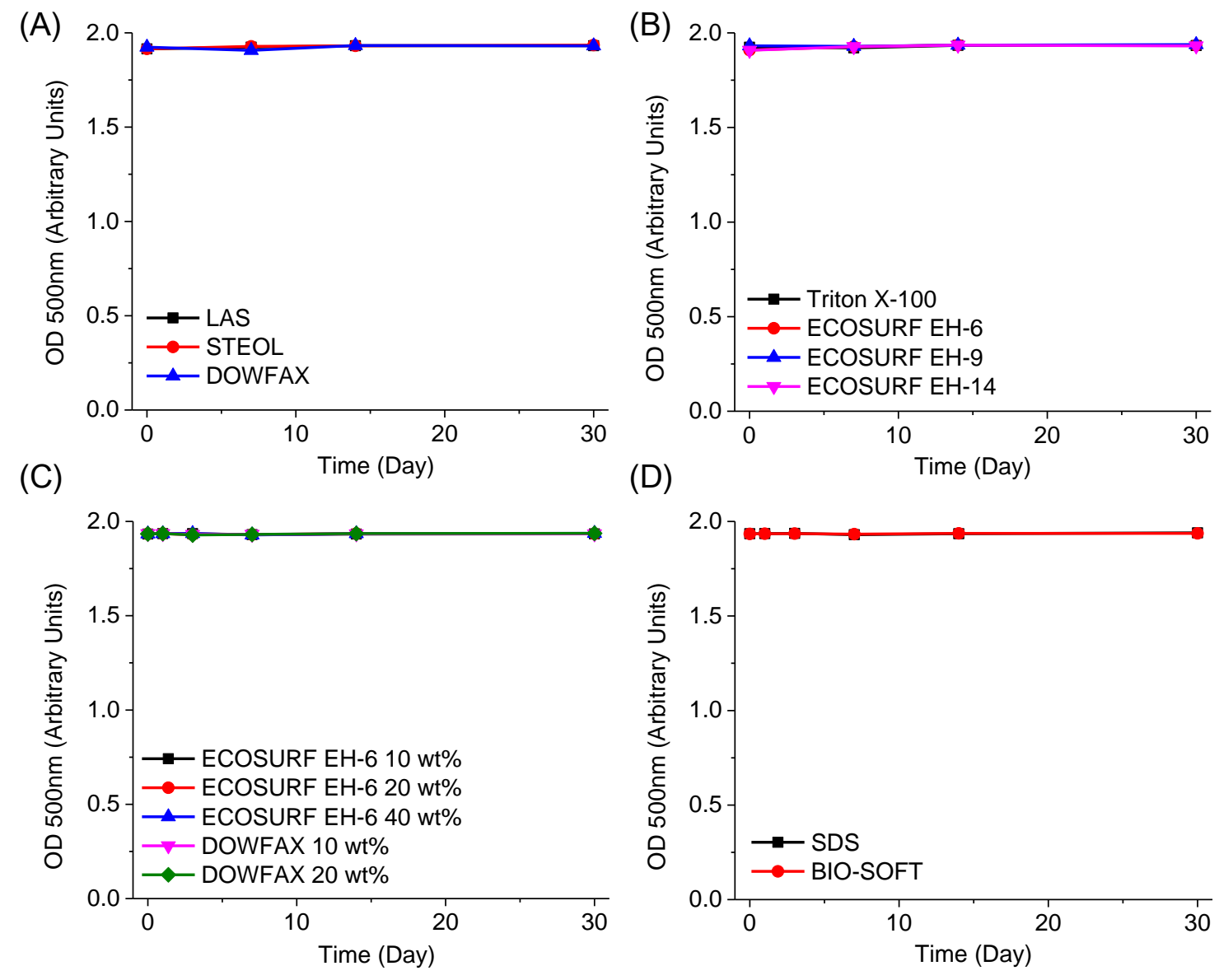

Figure S6. Turbidity tests at $500 \mathrm{~nm}$ of PEO- $b$-PSt vesicles in the presence of (A) various anionic surfactant at $5 \mathrm{wt} \%$; (B) various nonionic surfactant at $5 \mathrm{wt} \%$; (C) selected anionic and nonionic surfactants (ECOSURF EH-6 and DOWFAX) at higher concentration of 10, 20, and $40 \mathrm{wt} \%$; (D) selected anionic and nonionic surfactant (SDS and BIO-SOFT) at $5 \mathrm{wt} \%$ with shear force pretreatment (probe sonicator, $700 \mathrm{~W}, 50 \%$ amplitude, 14-s pulse sequence, 2-s pulse and 1-s delay). 


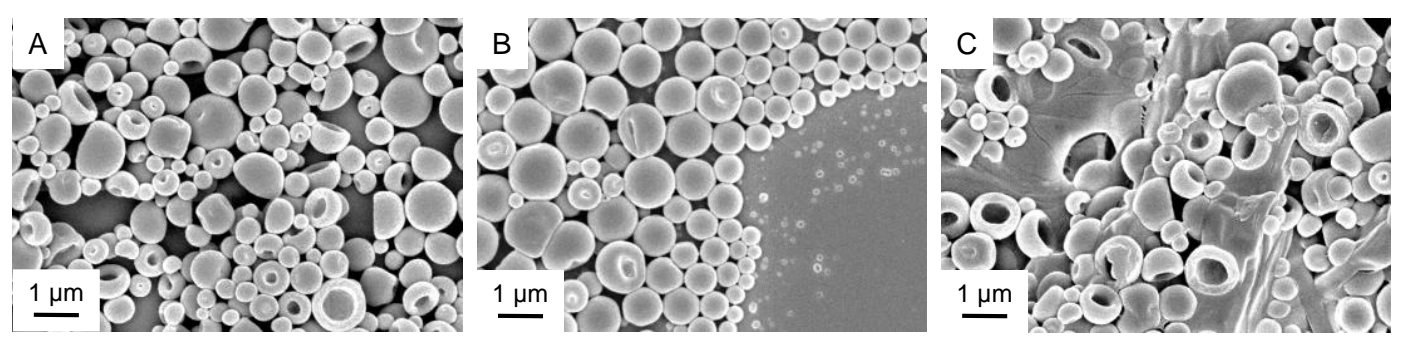

Figure S7. SEM images of polymersomes (A) before surfactant treatment; (B) after treatment with BIO-SOFT (10 wt\%); (C) after treatment with SDS (10 wt\%).
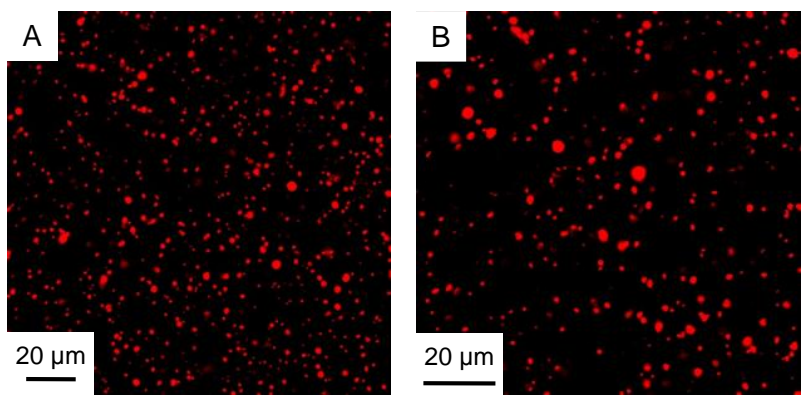

Figure S8. Confocal microscopy images of Rhodamine B (RhB) loaded $\mathrm{PEO}_{113}-b-\mathrm{PSt}_{1441}$ polymersomes.
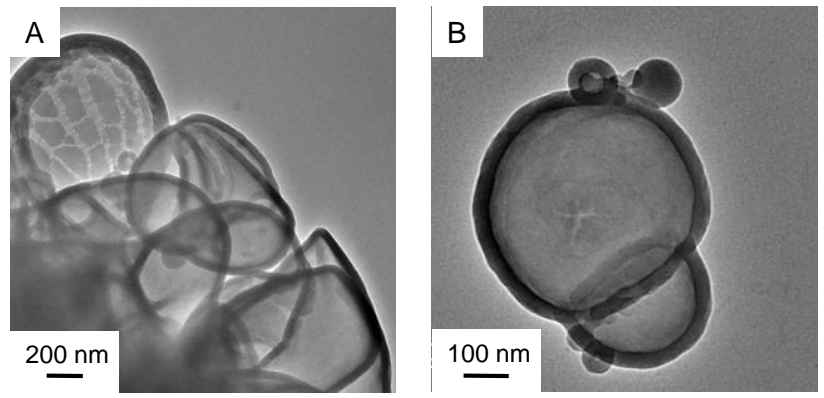

Figure S9. TEM images of $\mathrm{PEO}_{20}-b-\mathrm{PSt}_{250}$ polymersomes with thinner membranes. 


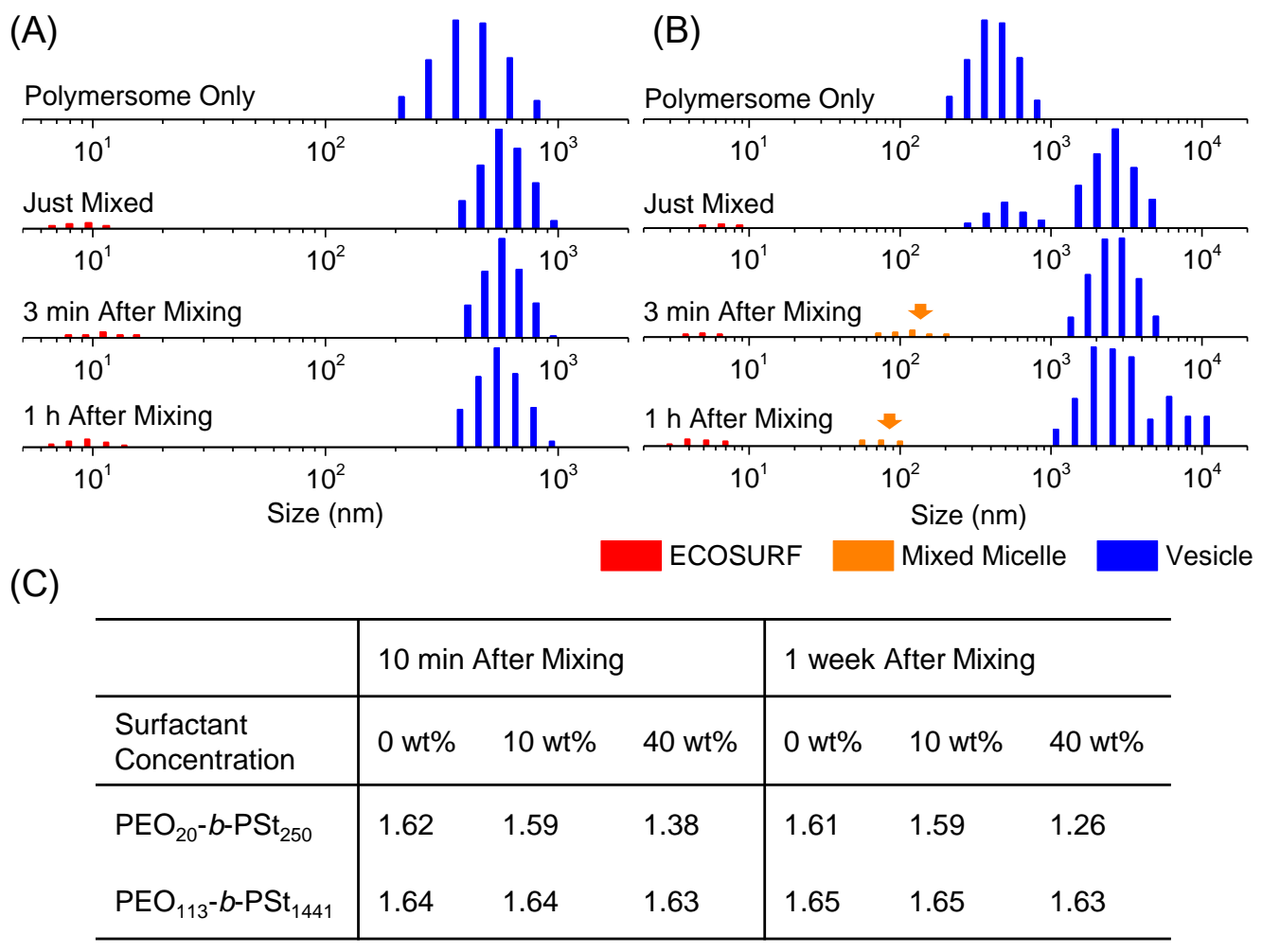

Figure S10. Surfactant resistance of $\mathrm{PEO}_{20}-b-\mathrm{PSt}_{250}$ polymersomes with thinner membranes. (A-B) DLS results (size vs intensity) of vesicles incubated with $10 \mathrm{wt} \%$ (A) and $40 \mathrm{wt} \%$ (B) ECOSURF EH-6 after various times. (C) Turbidity tests at $500 \mathrm{~nm}$ of $\mathrm{PEO}_{20}-b$ - $\mathrm{PSt}_{250}$ and $\mathrm{PEO}_{113}-b$-PSt ${ }_{1441}$ polymersomes in the presence of ECOSURF EH-6 at different concentrations.

From DLS results, $\mathrm{PEO}_{20-} b$ - $\mathrm{PSt}_{250}$ polymersomes was stable against $10 \mathrm{wt} \%$ surfactant, but partially solubilized by $40 \mathrm{wt} \%$ surfactant as verified by the appearance of new mixed surfactant-polymer micelles (indicated by orange arrow in Figure B). The partial polymersome disassembly was also demonstrated by the rapid optical density decrease at $500 \mathrm{~nm}$ in the presence of $40 \mathrm{wt} \%$ ECOSURF EH- 6 . The turbidity decrease was obvious only 10 min after mixing, and kept decreasing after 1 week incubation. As a comparison, no significant turbidity change was observed for $\mathrm{PEO}_{20}-b-\mathrm{PSt}_{250}$ polymersomes with 10 wt $\%$ surfactant treatment as well as $\mathrm{PEO}_{113}-b-\mathrm{PSt}_{1441}$ polymersomes incubated with 40 wt $\%$ surfactant. 


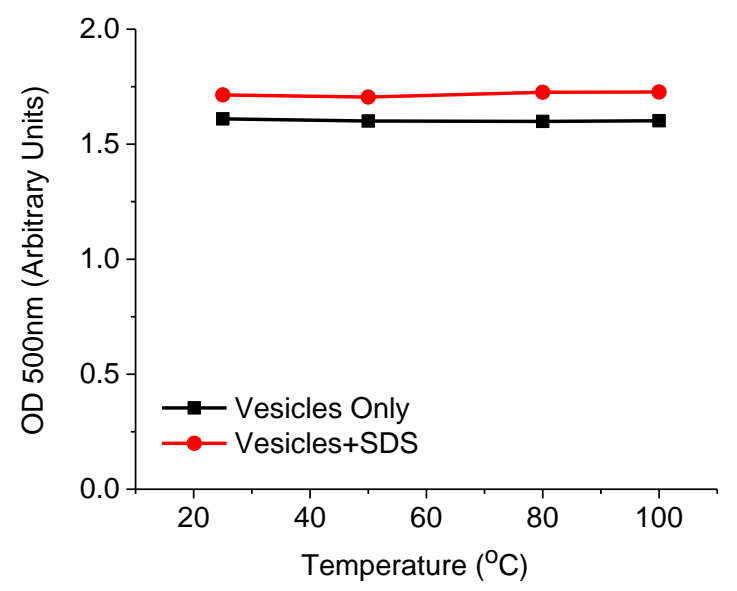

Figure S11. Turbidity tests at $500 \mathrm{~nm}$ of $\mathrm{PEO}_{113}-b-\mathrm{PSt}_{1441}$ polymersomes at different temperature in the absence and in the presence of SDS (10 wt $\%)$.

The aqueous suspension of polymersomes (with or without SDS) was placed in a sealed vial and heated to the target temperature $\left(50,80,100^{\circ} \mathrm{C}\right)$ for $1 \mathrm{~min}$. The suspension was then cooled down to rt (to avoid any temperature effect on turbidity such as PEO dehydration) and its turbidity at $500 \mathrm{~nm}$ was tested by UV-Vis spectrometer. The unchanged turbidity indicated that the polymersomes are stable against short time heating treatment even in the presence of surfactants. 